\title{
Does species richness drive speciation? A reassessment with the Hawaiian biota
}

\author{
Daniel S. Gruner, Nicholas J. Gotelli, Jonathan P. Price and Robert H. Cowie
}

D. S. Gruner(dsgruner@umd.edu),Bodega Marine Lab and Section of Evolution and Ecology, Univ. of California, Davis, CA 94923, USA (present address: Dept of Entomology, Univ. of Maryland, 4112 Plant Sciences Building, College Park, MD 20742, USA). - N. J. Gotelli, Dept of Biology, Univ. of Vermont, Burlington, VT 05405, USA. - J. P. Price, Dept of Geography and Environmental Studies, Univ. of Hawai i at Hilo, Hilo, HI 96720, USA. - R. H. Cowie, Center for Conservation Research and Training, Univ. of Hawai $i$, Honolulu, HI 96822, USA.

\section{Does richness cause speciation?}

After MacArthur and Wilson (1967), island biogeographical research focused primarily on processes of colonization and extinction over ecological time scales, but recent work has rejuvenated interest in the generation of diversity in situ over evolutionary time (Lomolino 2000, Losos and Schluter 2000, Gillespie 2004, Cadena et al. 2005). In a recent contribution, Emerson and Kolm (2005) proposed a causal link between species richness and speciation on islands. They suggested that species richness per se might increase the rate of speciation by intensifying competition and suppressing population densities of resident species, thereby allowing for increased genetic drift, greater lability in traits, and increased evolutionary change (Erwin 2005). Alternatively, increased structural complexity and heterogeneity associated with higher richness may stimulate niche diversification and speciation (Tokeshi 1999).

Emerson and Kolm (2005) supported their proposition with stepwise multiple regression analyses of island species richness for plants and arthropods in the Canary and Hawaiian archipelagos. For these analyses they proposed that the proportion of an island's biota that consisted of single-island endemics - hereafter "SIEs" - could be interpreted as an index of speciation. Their arcsinetransformed index was positively correlated with total island richness in each of their four analyses (plants and arthropods in the Canary and Hawaiian Islands). Emerson and Kolm (2005) therefore concluded that species richness itself may be an important causal driver of additional speciation and endemism in these oceanic archipelagos ("diversity-driven speciation hypothesis").

This proposal generated considerable controversy in the literature. Several rejoinders argued for alternative mechanisms as sufficient but more parsimonious explanations for observed endemism patterns on oceanic islands. Cadena et al. (2005) proposed that the accumulation of endemic species depends primarily on the realized lifespan of islands: older islands have both more opportunity to receive colonists and longer duration in which to accumulate endemics. With their "island immaturity - speciation pulse" hypothesis, Whittaker et al. (2007) extended this idea to consider ancient, senescent islands, where the eventual collapse of endemism and richness is observed as islands disappear beneath the sea surface. Pereira et al. (2007) also argued for the primacy of physical variables and time, but further demonstrated the insufficiency of multiple regression analyses as a test of this hypothesis. They showed that total richness and endemism patterns strongly covary with island size, elevation, distance to nearest island, and island age, and with richness of the other taxon (e.g. arthropods for plants) such that it is impossible with this analytical method to ascribe ultimate causation to withintaxon richness. Finally, several authors proposed null models to account for island endemism patterns attributable to statistical properties of random samples (Witt and Maliakal-Witt 2007, Kiflawi et al. 2007, Birand and Howard 2008). Emerson and Kolm (2007a, b) proposed a different (and we argue, inappropriate) null model in response to these criticisms.

In this paper, we revisit the relationship between endemism and species richness in a reanalysis of arthropod (Nishida 2002) and plant (Price 2004) data for the Hawaiian Islands and include a new dataset for land snails (Cowie et al. 1995, Cowie and Holland 2006). We propose distinct null models for endemism patterns in these island datasets. Because the methodological contrasts and findings of these different null models are more clear in the context of our assumptions, methods, and results, we address the specific null models of Emerson and Kolm (2007a, b), Witt and Maliakal-Witt (2007), Kiflawi et al. (2007), and Birand 
and Howard (2008) following the discussion of our analyses.

Emerson and Kolm's (2005) original regression analysis rested on the implicit assumption that, in the absence of driving speciation mechanisms, the number or proportion of endemic species should be independent of the total richness on individual islands (this assumption was later made explicit, Emerson and Kolm 2007a). Under this assumption, the statistical null hypothesis is of no correlation between the proportion of SIEs and total species richness. However, a universal property of biodiversity sampling curves is that rare species are disproportionately under-represented in small samples (Gotelli and Colwell 2001). At the biogeographic scale, we expect the proportion of SIEs to escalate with total species richness, even in the absence of mechanisms promoting in situ speciation. Therefore, we formulated two null sampling hypotheses that incorporated the expected non-linearity in SIE proportion with variation in the richness of island species pools.

\section{Null models for island endemism}

\section{Model 1: simple random sampling}

To investigate this null sampling hypothesis, we analyzed arthropod and plant data $(\mathrm{n}=17)$, and snail data $(\mathrm{n}=14 ; 3$ islands with zero native snails) for the Hawaiian islands obtained as species by island incidence matrices from original sources (Cowie et al. 1995, Nishida 2002, Price 2004, Cowie and Holland 2006). In contrast to Witt and Maliakal-Witt (2007), we used only confirmed records for native species for all taxa (total arthropod $S=5362$; plants $=1009$; snails $=732$ ). For each island, we simulated a random draw of species (without replacement) in which the probability of occurrence of each species was proportional to the number of islands it occupied in the archipelago. From the archipelago species lists, we sampled the observed number of species for each island and recorded the number classified as SIEs in the simulation. We repeated this procedure independently 1000 times for each island, generating the mean number of SIEs and true 95\% confidence interval. We then plotted the expected proportions of SIEs (arcsin transformed, sensu Emerson and Kolm 2005) and 95\% confidence intervals generated by the null model against taxon total species richness and compared these expectations to the observed data.

Simulations were executed in the $\mathrm{R}$ statistical package $<$ http://www.r-project.org/ $>$, code is provided in Supplementary material.

This null model makes the following assumptions: 1) species occurrences on islands are random and independent of one another; 2 ) the probability that a species occurs on an island is proportional to its observed number of occurrences in the archipelago; 3) the predicted species present on each island represent a random draw from the set of species in the archipelago; 4) total species number in each null assemblage is the same as on each real island; and 5) the detection probabilities for SIE versus non-SIE species are the same on all islands. Although the null model assumes that the species found on an island are "sampled" from an archipelago-wide source pool, the null model does not assume there are any differences in sampling intensity or efficiency on islands of different size. Instead, the model is based on the premise that the observed number of species and the observed number of SIEs that have been recorded on these islands are reasonable estimates of the true numbers. This premise also forms the basis for the original regression analysis (Emerson and Kolm 2005). This null model is a form of rarefaction (Gotelli and Colwell 2001); it was first used in biogeography almost a century ago to examine taxonomic ratios (Järvinen 1982) and it remains a standard sampling model for biodiversity indices (Enquist et al. 2002).

\section{Model 2: fixed-fixed model}

To ensure that our results were not sensitive to the specific details of our null model, we used a second randomization algorithm to generate the expected frequency of SIEs with random sampling. Instead of taking a random subsample to generate a species list for each island independently, we use a randomization method to simultaneously generate the species list for all the islands in the archipelago. The data are treated as a binary presence-absence matrix, in which the rows are species and the columns are sites (McCoy and Heck 1987). In this model, we created random matrices but retained the row sums ( $=$ number of species occurrences) and the column sums ( $=$ number of species per island) of the original matrix. This "fixed-fixed" null model (Gotelli 2000) has been widely used in studies of species cooccurrence (Gotelli and McCabe 2002), but also has been applied to studies of nestedness (Ulrich and Gotelli 2007), and phylogenetic structure (Webb et al. 2006), and its statistical properties have been well-studied (Gotelli and Entsminger 2001, Miklós and Podani 2004). The fixedfixed null model ensures that, in each null assemblage, all islands have the same number of species as observed, and that all species have the same number of occurrences as observed. However, the composition of species on each island (and hence the potential frequency of endemism) varies randomly within these constraints. Thus, in Model 2, each SIE can be placed on only one island in the archipelago. In Model 1, the same SIE could theoretically be placed on more than one island if it was sampled by chance. Model 1 (the simple random sampling model) is appropriate for comparing each island independently to a common source pool, whereas Model 2 (the fixed-fixed model) treats the entire archipelago as the sample, so that occurrences on each island do depend on the rest of the islands in the assemblage.

For plants and snails, 1000 random matrices for Model 2 were generated with EcoSim software ver. 7.72 (Gotelli and Entsminger 2004). Computational constraints prohibited running Model 2 for the arthropod matrix.

\section{Results and discussion}

For most islands, the observed arcsine-transformed proportion of SIEs was less than or equal to the indices predicted by Model 1 (Fig. 1A; arthropods: 13 of 17 islands; plants: 9 of 17 islands; snails: 7 of 14 islands). These results appear to 
A Model 1: simple random sampling

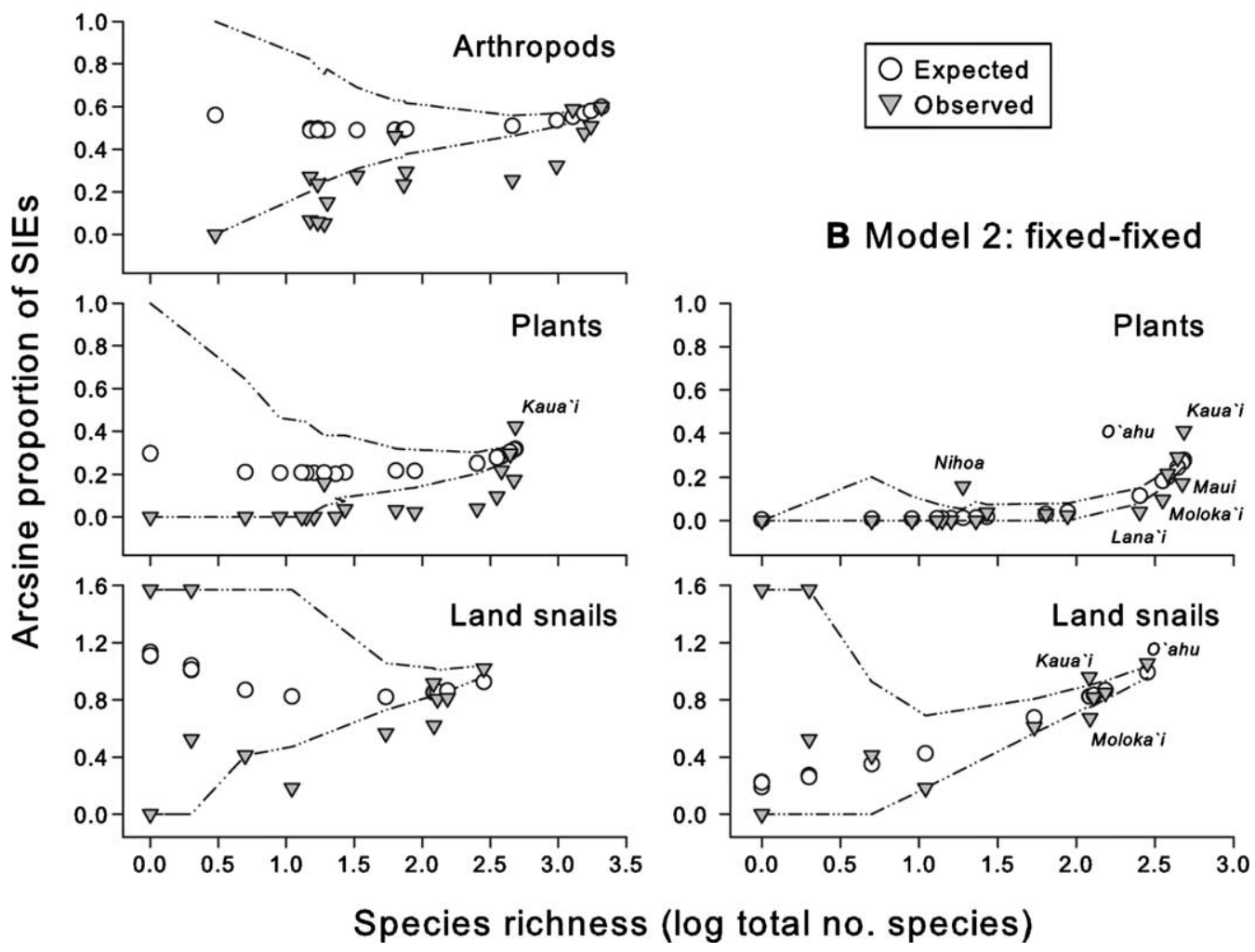

Fig. 1. Bivariate plots for the relationship between the arcsine-transformed proportion of single island endemics (SIEs) (Emerson and Kolm's [2005] "index of speciation") and the $\log _{10}$ total native species richness in Hawaiian arthropods, plants, and land snails generated from (A) Model 1 (simple random sampling) and (B) Model 2 (fixed-fixed model) simulations. Expected values from null model simulations are open circles, observed values are shaded triangles. Dashed lines represent the 95\% CI around the expected random sampling distribution. In Model 1 simulations (A), only plants on Kaua' $i$, out of all islands for the three taxa, had more SIEs than expected from random sampling (observed index $=0.422$, expected number $=0.316,95 \% \mathrm{CI}=0.285 / 0.346$ ). For many islands, the proportion of SIEs actually fell on or below the lower $95 \%$ confidence interval (13/17 for arthropods, $9 / 17$ for plants, $7 / 14$ for land snails). In Model 2 simulations (B), the observed indices from the islands of Kaua' $\mathrm{i}$ and $\mathrm{O}^{\prime}$ ahu were above the upper confidence interval for both snails and plants, and for plants on Nihoa; observed indices fell below expectation for plants on Lana' $i$, Moloka $i$ and Maui and for snails on Moloka'i.

contradict the hypothesis that diversity per se drives speciation, although they may have been affected by the possibility of randomly sampling the same SIE species by replacement. For the plant and arthropod data sets, the expected value of the index in the Model 1 simulations remained roughly constant across most of the range in observed richness, then increased gradually as larger random samples accumulated a greater fraction of rare taxa (Fig. 1A). For snails, the expected values fell initially, but this reflects the extremely low richness on these islands $(<5$ native species on $7 / 14$ islands), which distorts the ratios and also leads to wide confidence intervals.

Similar results were obtained with Model 2 (Fig. 1B); however, two clear differences emerge. When the model is constrained not merely to the same richness total per island (assumption 4, above), but also the occurrence frequency of each species is fixed to that observed in the dataset, the confidence intervals adhered more narrowly and the slopes of the expected values steepened in the upper tails of richness (Fig. 1). Endemism on most islands remained within or below the $95 \%$ confidence intervals, suggesting the observed arcsine-transformed index of speciation was generally weaker or within expectation from random sampling. However, Kaua $i$, the richest island for plants, contained more SIE plants than expected by chance in both simulations, and contained more SIE snails than expected with Model 2 simulations. The index for $\mathrm{O}^{\prime}$ ahu, richest for arthropods and snails and second richest for plants, fell within the $95 \%$ confidence intervals for all three taxa in Model 1, but emerged above upper confidence intervals for snails and plants in Model 2, along with Nihoa for plants.

As predictable from geological age-accumulation hypotheses (Willis 1922, Fischer 1960, Cadena et al. 2005, Whittaker et al. 2007), the highest endemism proportions were found on the oldest high islands in the geographic center of the archipelago ( $\mathrm{O}^{\prime} \mathrm{ahu}$, Kaua $\mathrm{i}$ ) for all three taxonomic groups (Fig. 1). Consistent with the "island immaturity - speciation pulse" hypothesis (Whittaker et al. 2007), independent syntheses from plant lineage data suggest that trends of phylogenesis and species accumulation reach their peak on the largest, youngest island (Hawai $i$ ), decline with increased local species packing on older large islands (e.g. Kaua i $\mathrm{i}$ ), and dwindle to zero on eroded and subsiding northwestern islets and atolls (Sakai et al. 1995, Price 2004). By contrast, Maui, Moloka 'i, and Lana' $i$ of the Maui Nui complex were joined as one land 
mass only $20000 \mathrm{yr}$ ago (Price and Elliot-Fisk 2004). We would expect that the recent connectance of this island group would enhance dispersal and genetic introgression among divergent races, thereby driving the observed SIEs of these islands below the null model expectations (Fig. 1B). On balance, the few departures from random sampling are consistent with phylogenetic and community ecological work that implicates island size and geological history as the ultimate drivers of evolutionary species accumulation in the Hawaiian Islands (Cowie 1995, Gillespie 2004, Price 2004, Cadena et al. 2005, Gruner 2007, Whittaker et al. 2007).

The slopes of arcsin-transformed index versus species richness relationships appear somewhat steeper at the richest tails of the observed versus the simulated Model 1 data (Fig. 1). ANCOVA analysis of these relationships grouped by the observed versus expected island endemism indices as a categorical covariate showed a significant interaction between total richness and the covariate for arthropods and plants, but not snails (Table 1). These subtle patterns could perhaps be interpreted as support for diversity-driven speciation hypothesis. However, the two linear regressions of the observed and expected indices cross each other at island richness totals that are larger than the most speciesrich island observed for each taxon in the Hawaiian archipelago (Fig. 2; arthropod total richness $=7161$, plants $=5137$, snails $=907$ ). In other words, even this result cannot account for the observed pattern of endemism in the richness range of the Hawaiian archipelago.

The regression analysis presented by Emerson and Kolm (2005), questioned already for its power to demonstrate causation (Pereira et al. 2007), presents the endemism index as a ratio of the form $\mathrm{y} / \mathrm{x}$ regressed against $\mathrm{x}$ (SIEs/total species number versus total species number). Although Emerson and Kolm (2005) ran diagnostics to evaluate the independence of their richness variables, Brett (2004) cautioned that regressions incorporating predictors in the response variables can lead to spurious statistical correlations. The arcsine-transformation of the proportional index introduces additional distortion in observed and expected values at small sample sizes (snails, Fig. 1), and the assumption of normal error distribution for the parametric regression model can be violated. The null models that we have used here directly account for these possibilities because randomization methods do not depend on the assumption of normality, and they take into account the non-independence of $y / x$ when regressed against $x$. A more natural index is simply the observed number of SIEs per island. When the data are plotted as observed and expected number of SIEs on $\log -\log$ axes, the emergent result remains: the number of SIE taxa found on most islands is either about the same or slightly less than would have been expected by chance (Fig. 2). ANCOVA analysis applied to log-transformed total SIE showed no difference in the slopes of expected and observed relationships for plants and snails, and a weak interaction for arthropods with convergence beyond the observed richness of any island (Fig. 2, Table 1).

\section{Other null models}

Several recent papers have also used null model methodology to address the problem of island endemism. Witt and Maliakal-Witt (2007) argue that it is a tautological property that two lists of island species with shared and unshared elements will always have a greater proportion of endemics on the more species-rich island. When the argument is extended to $\mathrm{N}$-species lists, it is possible to construct special cases that violate this inequality, but we agree with their general point that most random re-arrangements of island species lists will contain a greater proportion of endemics in larger faunas. Witt and Maliakal-Witt's (2007) null models (which they developed independently of our analysis) illustrate that point and are most similar to our Model 1 (simple random sampling), which also uses sampling with replacement, but does not restrict endemics to single occurrences. Our Model 2 (fixed-fixed) simultaneously preserves the occurrence frequency distribution and the

Table 1. ANCOVA results for the relationships between endemism and total richness in Hawaiian arthropods, plants and snails. ANCOVA models test the relationship of $\log _{10}$ native species richness and the group covariate with the (a) arcsine-transformed proportion of single island endemics (SIEs) (Emerson and Kolm's [2005] "index of speciation") and (b) $\log _{10}$ richness of SIEs. The covariate was defined by whether the datapoint was an observed or expected value generated from null Model 1 (random sampling) simulations.

\begin{tabular}{|c|c|c|c|c|c|c|c|}
\hline \multirow[t]{2}{*}{ Source } & \multicolumn{4}{|c|}{ (a) asin [SIEs/total S] } & \multicolumn{3}{|c|}{ (b) $\log _{10}[\mathrm{SIEs}+\mathrm{I}]$} \\
\hline & DF & SS & $\mathrm{F}$ & $\mathrm{p}$ & SS & $\mathrm{F}$ & $\mathrm{p}$ \\
\hline \multicolumn{8}{|l|}{ Arthropods } \\
\hline $\log _{10}$ total richness & 1 & 0.2969 & 51.522 & $<0.0001$ & 30.7146 & 1717.63 & $<0.0001$ \\
\hline Group & 1 & 0.5781 & 100.314 & $<0.0001$ & 0.7086 & 39.629 & $<0.0001$ \\
\hline Richness $\times$ group & 1 & 0.1285 & 22.304 & $<0.0001$ & 0.1863 & 10.417 & 0.003 \\
\hline Error & 30 & 0.1729 & & & 0.5365 & & \\
\hline \multicolumn{8}{|l|}{ Plants } \\
\hline $\log _{10}$ total richness & 1 & 0.1001 & 20.039 & 0.0001 & 17.7496 & 185.498 & $<0.0001$ \\
\hline Group & 1 & 0.2055 & 41.142 & $<0.0001$ & 1.4446 & 15.0977 & 0.0005 \\
\hline Richness $\times$ group & 1 & 0.0309 & 6.180 & 0.0187 & 0.0192 & 0.2000 & 0.6576 \\
\hline Error & 30 & 0.1498 & & & & & \\
\hline \multicolumn{8}{|l|}{ Snails } \\
\hline $\log _{10}$ total richness & 1 & 0.0009 & 0.008 & 0.9319 & 18.4592 & 926.790 & $<0.0001$ \\
\hline Group & 1 & 0.8048 & 6.292 & 0.0193 & 0.1306 & 6.5586 & 0.017 \\
\hline Richness $\times$ group & 1 & 0.1995 & 1.560 & 0.2238 & 0.0147 & 0.7383 & 0.398 \\
\hline Error & 24 & 3.0700 & & & & & \\
\hline
\end{tabular}




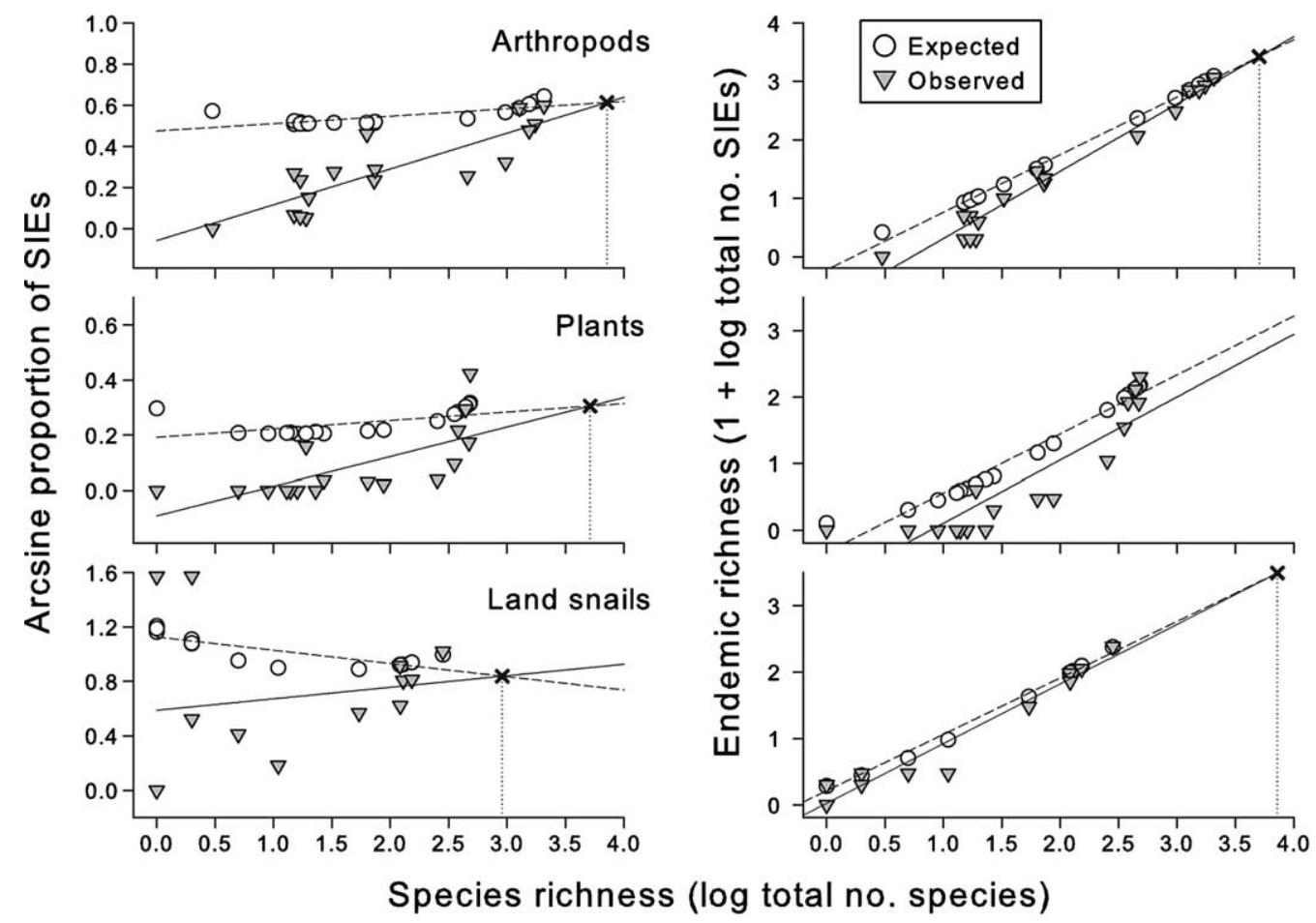

Fig. 2. Bivariate plots for relationships between endemism and the $\log _{10}$ total native species richness in Hawaiian arthropods (top panels), plants (middle), and land snails (bottom). The left-hand panels show the arcsine-transformed proportion of SIEs and the righthand panels show $\log _{10}$ transformed total SIEs. Expected values from the Model 1 simulation are open circles, observed values are shaded triangles. Univariate linear regressions are plotted with dashed lines for expected values and solid lines for observed values. The relationship with observed values of the index was not significant for snails $\left(R^{2}=0.0279, D F=12, p=0.568\right)$. The points at which expected relationships would cross with observed relationships are marked with an $\mathrm{X}$ and dropline (arcsine index: arthropod total richness $=7161$, plants $=5137$, snails $=907 ; \log$ total no. SIEs: arthropod total richness $=5058$, plants $=3.3 \times 10^{7}$ [not shown], snails $=7244$ ).

observed species richness per island, ensuring that the number of endemic species in the simulated archipelago exactly matches the number in the real data.

In their critiques of the null models of Witt and Maliakal-Witt (2007) and Kiflawi et al. (2007), Emerson and Kolm $(2007 \mathrm{a}, \mathrm{b})$ offer an alternative null model that was based on our freely available EcoSim software (Gotelli and Entsminger 2004). Emerson and Kolm (2007a: 336) stated that: "we have carried out simulations maintaining observed values for the number of species per island, but allowing species within an island to 1) go locally extinct, 2) successfully colonise another island, or 3) undergo either anagenetic or cladogenetic speciation. Importantly these events are random, with local extinction being balanced by either colonisation or speciation to maintain species number."

However, the EcoSim randomization software produces classical null model tests, which are stochastic randomizations of observed data that do not incorporate specific biological processes (Gotelli and Graves 1996). EcoSim algorithms do not include any explicit processes of extinction, colonization, or speciation. In some cases, mechanistic models such as the neutral model can be interpreted as a kind of null model (Gotelli and McGill 2006), but the claim (Emerson and Kolm 2007a) that these models include extinction, colonization and speciation processes is incorrect. In contrast, both Witt and Maliakal-Witt (2007) and Birand and Howard (2008) presented null models in which they explicitly simulated random colonization and extinction processes, and they largely reproduced the empirical patterns in Emerson and Kolm (2005).

The EcoSim software offers several possible algorithms for randomizing occurrence matrices (Gotelli and Entsminger 2001). Emerson and Kolm (2007a, b) unfortunately chose the "fixed columns, equiprobable rows" option for their analyses. This model preserves the number of species per site, but it relaxes the row totals of the matrix and assumes that occurrence frequencies of all species are equiprobable. The equiprobable model for species means that, on average, all species are assigned the same number of occurrences. Thus, the simulated frequency of endemism in the archipelago will be artificially inflated because widespread species will occur less frequently and endemic species will occur more frequently in the null communities compared to the original data. The net effect will be to reduce the expected frequency of endemics for every island.

This flaw in their model is readily apparent in figures plotting their results: for every island in the archipelago, the observed assemblage always had more endemics than the simulated assemblages (Fig. 2 of Emerson and Kolm 2007a, Fig. 3 of Emerson and Kolm 2007b). But if the frequency of endemism in the archipelago were preserved, then an excess of endemics on some islands must be balanced by a deficit on other islands. This is exactly what we see from our models (Fig. 1), from other published models (Kiflawi et al. 2007, Birand and Howard 2008), 
and would also expect from Witt and Maliakal-Witt's (2007) framework. Because the null model of Emerson and Kolm (2007a, b) seems flawed, their slope test does not provide valid support for their hypothesis that species richness promotes endemism.

\section{Conclusions}

There is little debate that in situ speciation is a dominant process generating biological diversity on oceanic islands (Darwin 1859, Grant 1998, Whittaker 1998). In general, more research is required to determine which factors stimulate speciation, and the "diversity-driven speciation" hypothesis is one possibility that deserves further attention. However, such efforts should state clear null hypotheses that can distinguish a robust increase in SIE proportion with richness from a spurious result generated by sampling. Emerson and Kolm (2005: 1017) write of an "overlooked prediction of island biogeographic theory: as species number in an area increases, so should the rate of speciation." As demonstrated by our analyses, however, a positive relationship can be expected simply from the sampling properties of species pools. We found consistent results using data for three different assemblages (arthropods, snails, plants), with two different null models (random sampling, and fixed-fixed) and two indices of speciation (arcsine-transformed index and the number of SIEs per island). Of course, both the slope and the observed level of endemism will be sensitive to the details of null model construction and the assumptions of random sampling and species independence. Nevertheless, our analyses align fundamentally with other published models incorporating different assumptions and analytic techniques (Witt and Maliakal-Witt 2007, Kiflawi et al. 2007, Birand and Howard 2008). These analyses, individually and in sum, confirm that a positive correlation between species richness and the proportion of SIEs by itself is insufficient evidence to support the diversity-driven speciation hypothesis.

Acknowledgements - We thank Rob Colwell, Don Strong, Tad Fukami, Alexandre Diniz-Filho, Brent Emerson, and two anonymous reviewers for useful critical comments, and Rich Pyle and the Hawaii Biological Survey, Bishop Museum, Honolulu, for access to data. NJG acknowledges the support of NSF grant 0541936.

\section{References}

Birand, A. and Howard, D. J. 2008. The relationship between proportion of endemics and species diversity on islands: expectations from a null model. - Ecography 31: 286-288.

Brett, M. T. 2004. When is a correlation between nonindependent variables "spurious"? - Oikos 105: 647-656.

Cadena, C. D. et al. 2005. Is speciation driven by species diversity? - Nature 438: E1-E2.

Cowie, R. H. 1995. Variation in species diversity and shell shape in Hawaiian land snails: in situ speciation and ecological relationships. - Evolution 49: 1191-1202.

Cowie, R. H. and Holland, B. S. 2006. New island records for the endemic Hawaiian land snail Succinea caduca (Mighels 1845). - Bishop Mus. Occas. Pap. 88: 58-60.
Cowie, R. H. et al. 1995. Catalog of the native land and freshwater molluscs of the Hawaiian Islands. - Backhuys Publ.

Darwin, C. 1859. On the origin of species. - Murray.

Emerson, B. C. and Kolm, N. 2005. Species diversity can drive speciation. - Nature 434: 1015-1017.

Emerson, B. C. and Kolm, N. 2007a. Response to comments on Species diversity can drive speciation. - Ecography 30: 334338.

Emerson, B. C. and Kolm, N. 2007b. Species diversity can drive speciation: reply. - Ecology 88: 2135-2138.

Enquist, B. J. et al. 2002. General patterns of taxonomic and biomass partitioning in extant and fossil plant communities. - Nature 419: 610-613.

Erwin, D. H. 2005. Seeds of diversity. - Science 308: 1752-1753.

Fischer, A. G. 1960. Latitudinal variations in organic diversity. - Evolution 14: 64-81.

Gillespie, R. G. 2004. Community assembly through adaptive radiation in Hawaiian spiders. - Science 303: 356-359.

Gotelli, N. J. 2000. Null model analysis of species co-occurrence patterns. - Ecology 81: 2606-2621.

Gotelli, N. J. and Graves, G. R. 1996. Null models in ecology. - Smithsonian Inst. Press.

Gotelli, N. J. and Colwell, R. K. 2001. Quantifying biodiversity: procedures and pitfalls in the measurement and comparison of species richness. - Ecol. Lett. 4: 379-391.

Gotelli, N. J. and Entsminger, G. L. 2001. Swap and fill algorithms in null model analysis: rethinking the knight's tour. - Oecologia 129: 281-291.

Gotelli, N. J. and McCabe, D. J. 2002. Species co-occurrence: a meta-analysis of J. M. Diamond's assembly rules model. - Ecology 83: 2091-2096.

Gotelli, N. J. and Entsminger, G. L. 2004. EcoSim: null models software for ecology. - Acquired Intelligence and Kesey-Bear.

Gotelli, N. J. and McGill, B. J. 2006. Null versus neutral models: what's the difference? - Ecography 29: 793-800.

Grant, P. R. (ed.) 1998. Evolution islands. - Oxford Univ. Press.

Gruner, D. S. 2007. Geological age, ecosystem development, and arboreal arthropod community structure in the Hawaiian Islands. - Biol. J. Linn. Soc. 90: 551-570.

Järvinen, O. 1982. Species to genus ratios in biogeography: a historical note. - J. Biogeogr. 9: 363-370.

Kiflawi, M. et al. 2007. Species diversity can drive speciation: comment. - Ecology 88: 2132-2135.

Lomolino, M. V. 2000. Ecology's most general, yet protean pattern: the species-area relationship. - J. Biogeogr. 27: 17-26.

Losos, J. and Schluter, D. 2000. Analysis of an evolutionary species-area relationship. - Nature 408: 847-850.

MacArthur, R. H. and Wilson, E. O. 1967. The theory of iland biogeography. - Princeton Univ. Press.

McCoy, E. D. and Heck, K. L. Jr 1987. Some observations on the use of taxonomic similarity in large-scale biogeography. - J. Biogeogr. 14: 79-87.

Miklós, I. and Podani, J. 2004. Randomizations of presenceabsence matrices: comments and new algorithms. - Ecology 85: 86-92.

Nishida, G. M. (ed.) 2002. Hawaiian terrestrial Arthropod checklist. - Bishop Museum Press.

Pereira, H. M. et al. 2007. Does species diversity really drive speciation? - Ecography 30: 328-330.

Price, J. P. 2004. Floristic biogeography of the Hawaiian Islands: influences of area, environment and paleogeography. - J. Biogeogr. 31: 1-14.

Price, J. P. and Elliot-Fisk, D. 2004. Topographic history of the Maui Nui complex, Hawai $i$, and its implications for biogeography. - Pac. Sci. 58: 27-45.

Sakai, A. K. et al. 1995. Biogeographical and ecological correlates of dioecy in the Hawai' ian flora. - Ecology 76: 2530-2543. 
Tokeshi, M. 1999. Species coexistence: ecological and evolutionary perspectives. - Blackwell.

Ulrich, W. and Gotelli, N. J. 2007. Null model analysis of species nestedness patterns. - Ecology 88: 1824-1831.

Webb, C. et al. 2006. Phylocom: software for the analysis of community phylogenetic structure and character evolution. - < http://www.phylodiversity.net/phylocom/ > .

Whittaker, R. J. 1998. Island biogeography: ecology, evolution and conservation. - Oxford Univ. Press.

Download the Supplementary material as file E5289 from $<$ www.oikos.ekol.lu.se/appendix $>$.
Whittaker, R. J. et al. 2007. The island immaturity - speciation pulse model of island evolution: an alternative to the "diversity begets diversity" model. - Ecography 30: 321-327.

Willis, J. C. 1922. Age and area: a study in the geographical distribution and origin of pedes. - Cambridge Univ. Press.

Witt, C. C. and Maliakal-Witt, S. 2007. Why are diversity and endemism linked on islands? - Ecography 30: 331-333. 